\title{
Perhaps They are not Globular Clusters After All
}

\author{
A. M. Karick \\ School of Physics, University of Melbourne, Victoria 3010, Australia
}

\begin{abstract}
Our 2dF Fornax Cluster Spectroscopic Survey (FCSS) and followup work in the Virgo Cluster have shown that the cores of both galaxy clusters contain a previously-unknown class of object, ultra-compact dwarf (UCD) galaxies. We present high resolution spectroscopy and deep multicolor imaging to show that these enigmatic objects are dynamically distinct from both globular clusters (GCs) and nucleated dwarf galaxies (dE,Ns). Our hypothesis for their origin may explain the observed high "specific frequency" of GCs in central cluster galaxies.
\end{abstract}

\section{Dynamical Analysis and Stellar Populations}

Internal velocity dispersions of the UCDs and a comparison dE,N (FCC303) have been measured using the VLT and Keck Telescopes (Drinkwater et al. 2003). The velocity dispersions of the UCDs range from 24 to $37 \mathrm{~km} \mathrm{~s}^{-1}$, considerably higher than those of Galactic GCs. The UCDs lie well off the globular cluster $\mathrm{L} \propto \sigma^{1.7}$ relation in a previously unoccupied region. This supports the "galaxy threshing" model in which the UCDs are the remnant nuclei of infalling $\mathrm{dE}, \mathrm{Ns}$ which have been tidally disrupted by the cluster cD galaxy (Bekki et al. 2001). Removing the halo of the $\mathrm{dE}, \mathrm{N}$ galaxy reduces the total luminosity by about a factor of 100 but barely changes the central velocity dispersion. We obtained deep multicolour imaging $(\mathrm{u}, \mathrm{g}, \mathrm{r}, \mathrm{i})$ of the core of the Fornax Cluster taken with the CTIO MOSAIC camera. The colours of the UCDs were compared with a sample of NGC 1399 globulars (Mieske et al. 2002) and the nuclei of cluster $\mathrm{dE}, \mathrm{Ns}$. Preliminary analysis suggests that the UCDs have colours similar to the nuclei of the brightest $\mathrm{dE}, \mathrm{Ns}$ and GCs.

\section{Acknowledgements}

This work was done in collaboration with M. J. Drinkwater, M. D. Gregg and M. Hilker.

\section{References}

Bekki, K., Couch, W.J., Drinkwater, M. J., 2001, ApJ, 552, L105

Drinkwater, M. J., Gregg, M. D., Hilker, M., Bekki, K., Couch, W. J., Ferguson, H. C., Jones, J. B., Phillipps, S., 2003, Natur, 423, 519

Mieske, S. Hilker, M. Infante, L., 2002, A\&A, 383, 823 